\title{
Immunofluorescence Restaining of Biological Materials as a Viable Quantitative Procedure for Confocal Microscopy
}

\section{Z. Hesp and L. Isaacson}

Center for Neuroscience and Behavior, Department of Zoology, Miami University, Oxford, $\mathrm{OH}$ 45056

Immunofluorescence is a common technique used in light microscopy to visualize proteins and other small molecules primarily in biological tissue. This technique utilizes the principles of immunohistochemistry, where an endogenous molecule in the sample (the antigen) is tagged with a primary antibody specific for that molecule, which is in turn tagged with a secondary antibody that fluoresces within a certain wavelength range. There are some drawbacks however to this procedure, such as eventual photobleaching, delicacy of the tissue after sample preparation, and the inability to perform further analysis on the tissue after it has been stained and coverslipped. This can often pose an expense- and time-related problem when a sample has been stained for a given protein, but subsequent analysis of a different protein must be performed on an entirely new set of tissue.

Herein we demonstrate through confocal microscopy that previously stained tissue can be successfully 'restained' for a different protein, thereby preserving the tissue and reducing costs, time, and materials. In our model we examine the middle cerebral artery (MCA) in a rat, part of the extracerebral blood vessels on the base of the brain, for axonal density of tyrosine hydroxylase (TH), a marker for sympathetic axons. We compare the axonal density of TH axons in the MCA of control vessels (stained once for $\mathrm{TH}$ ) to the axonal density in treated vessels that are stained once for a different protein (vesicular acetylcholine transporter), coverslipped and imaged, and then restained for TH. Preliminary data, as shown in Figs. 1 and 2 (internal carotid artery), suggests that there is no statistically significant difference between the TH axonal density of the control vessels compared to that of the restained vessels when viewed with a confocal microscope.

While this data reflects more qualitative measurements, we show that for free-floating vessels the initial staining process does not degrade the tissue enough to prevent reuse, that other antigen binding sites are not blocked and are still viable, and that data derived from restaining may be equivalent to tissue only treated once. These are promising results, for it suggests that biological samples may be preserved for future use of additional immunofluorescence using confocal microscopy [1].

\section{References}

[1] This research was supported by NIH NS051206 to LGI. The aid of Dr. Richard Edelmann, Director of the Miami University Imagining Facility, is gratefully acknowledged. 


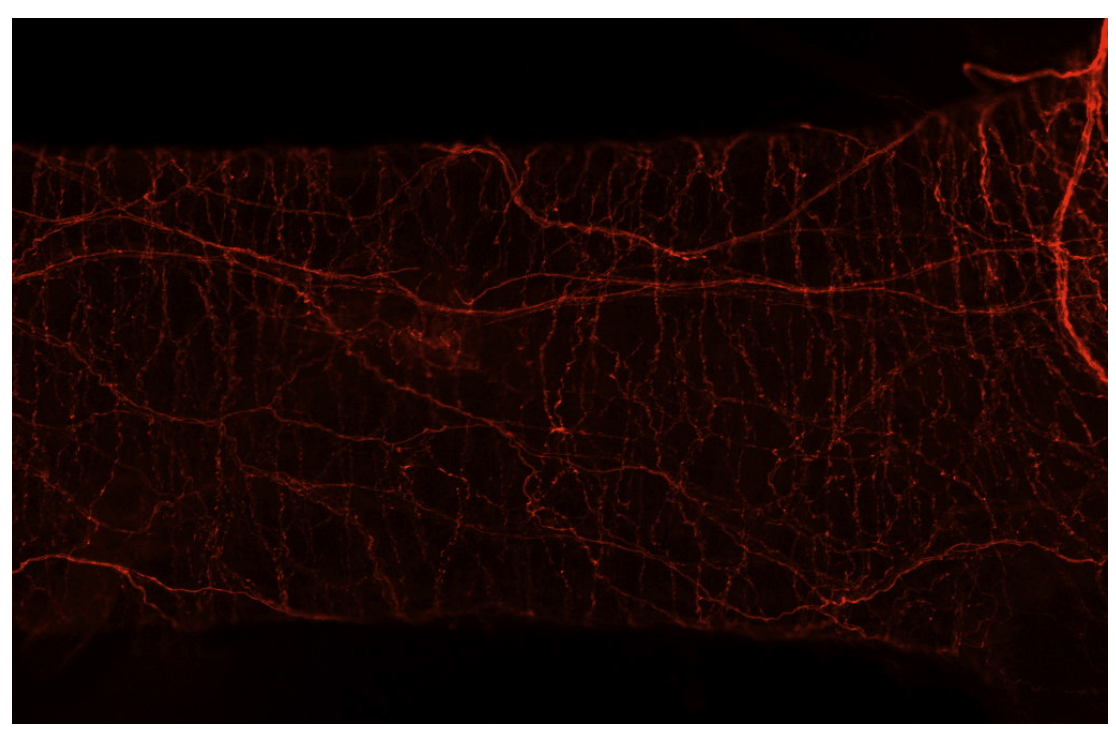

FIG. 1. Internal carotid artery (ICA) of the extracerebral blood vessels stained once for tyrosine hydroxylase. The ICA branches from the middle cerebral artery. Note the distinct axonal plexus and clarity of single axons verses bundles.

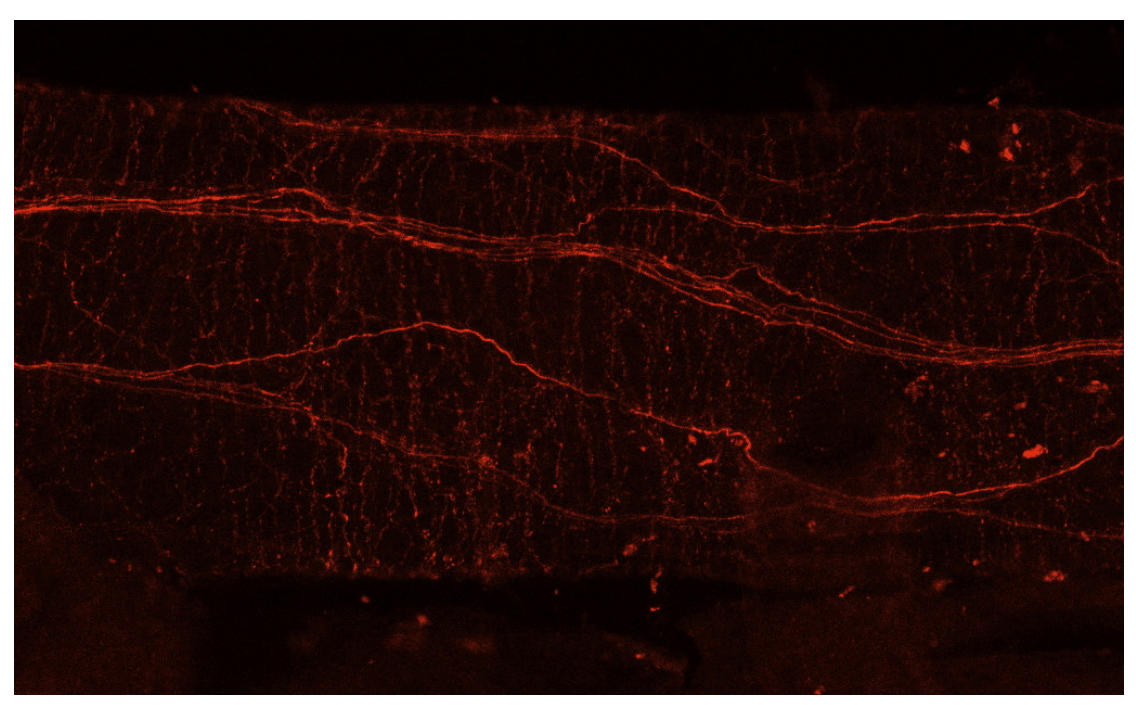

FIG. 2. Internal carotid artery of the extracerebral blood vessels stained for tyrosine hydroxylase a second time after initial staining with a different protein. The staining pattern is similar in organization and clarity as the vessel that was only stained once. 